\title{
Human-Robot Cooperative Swinging of Complex Pendulum-like Objects
}

\author{
Philine Donner, Franz Christange and Martin Buss
}

\begin{abstract}
This paper investigates human-robot cooperative swinging of a complex pendulum-like object. The complexity of the object results in two possible swinging modes. The goal is to excite one mode such that a desired energy level of the pendulum is reached while simultaneously damping the other mode. The energy based control concept relies on the projection of the complex mechanism onto an abstract simple pendulum with two-sided actuation. An actively contributing robot leader and robot follower are implemented. The controller performance is analyzed through simulations. A virtual reality experiment shows the transferability of the control approach to a human interaction partner.
\end{abstract}

\section{INTRODUCTION}

Robotics research has been directed towards bringing robots into everyday life. Consequently, robots need to be able to physically interact with humans, e.g. to cooperatively manipulate bulky objects. Cooperative object transport is one commonly studied scenario [1]-[3], in which a human-robot team moves a bulky object, e.g. a table, to a desired location. Besides these works on quasi-static object manipulation, only little focus has been paid towards dynamic object manipulation. An example scenario for cooperative dynamic object manipulation is the swinging of a sports mat onto a trolley by synchronized incremental energy injection of two partners (Fig. 1(b)). This scenario shows how swing motion extends the manipulation capabilities of a humanrobot team towards larger and heavier objects than they could manipulate on their own or through pure quasi-static motion.

Robotic example applications for swing motion are brachiating robots [4] and under-actuated cable-suspended robots [5]. To the best of the authors knowledge, the only works including swing-motion and physical interaction of a robot and a human have been on rope turning [6], [7]. Both papers limit their focus on sustaining a rope turning motion that was established by the human partner beforehand.

Figure 1 shows an illustration of flexible object swinging (b) as a combination of two extremes: an oscillating entity formed by the partners' arms and a rigid object (a) and a pendulum-like object that can oscillate itself (c). In [8], we investigated goal-directed cooperative swinging of a simple $\mathrm{v}$-shaped pendulum with a point mass in contrast to the cylindrical mass displayed in Fig. 1(c). An energy-based control concept based on [9] was developed that allows a robot and a human partner to cooperatively swing up the v-shaped pendulum to a desired energy level. The desired

P. Donner, F. Christange and M. Buss are with the Institute of Automatic Control Engineering, Technische Universität München, 80290 München, Germany, philine.donneretum.de, franz.christangedtum.de, mbetum.de

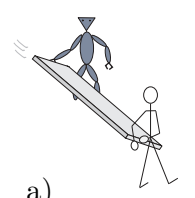

a)

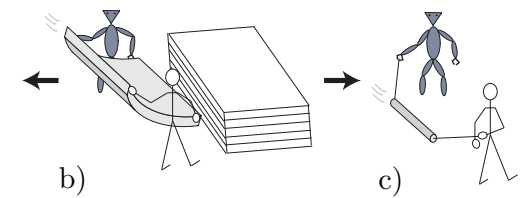

b)

c)
Fig. 1. Classification of flexible object swinging (b) as a combination of rigid object swinging (a) and pendulum swinging (c).

energy level can represent a desired object height at which the object could be released to perform a goal-directed throw. The robot can take on the roles of a leader and a follower based on whether the desired object energy is known to the robot in advance. The forces measured at the end effector are the only feedback the robot receives about the state of the object and the partner's intention. A virtual reality experiment showed that the robotic leader and follower yield similar results compared to their human counterparts.

In this paper, the control concept of [8] is extended to allow cooperative swing-up of more complex pendulum-like objects that can oscillate in several modes (see Fig. 1(c)). Our control approach relies on the projection of the complex pendulum on an abstract simple pendulum with two-sided actuation. While this projection is a simplification, simple pendulums have been successfully used to model various complex mechanisms, e.g. dance partners [10] or fluid in containers [11]. Within robotic walking research, leg dynamics are frequently modeled through spring-loaded linear inverted pendulums [12]. In [4] a two-link robot brachiates in ape-like fashion when being controlled to achieve pendulumlike dynamics.

In Sec. II we describe the complex pendulum-like object considered in this paper and give the problem statement. We introduce the abstract simple pendulum in Sec. III as the basis of the controller presented in Sec. IV. The controller performance is discussed based on simulation in Sec. V. A virtual reality experiment in Sec. VI shows the transferability of the presented controllers to a human interaction partner. We draw our conclusions in Sec. VII.

\section{PROBLEM FORMULATION}

\section{A. Setup and notation}

As an example pendulum that can swing in several modes we consider a trapezoidal pendulum as depicted in Fig. 2. We refer to it as a t-pendulum throughout this paper. The cylindrical pendulum object of mass $m_{\mathrm{p}}$, inertia $I_{\mathrm{p}}$ and length $l_{\text {cyl }}$ is suspended from two ropes, both of length $l$. The ropes themselves are connected to handles of mass $m_{\mathrm{h}}$. The 


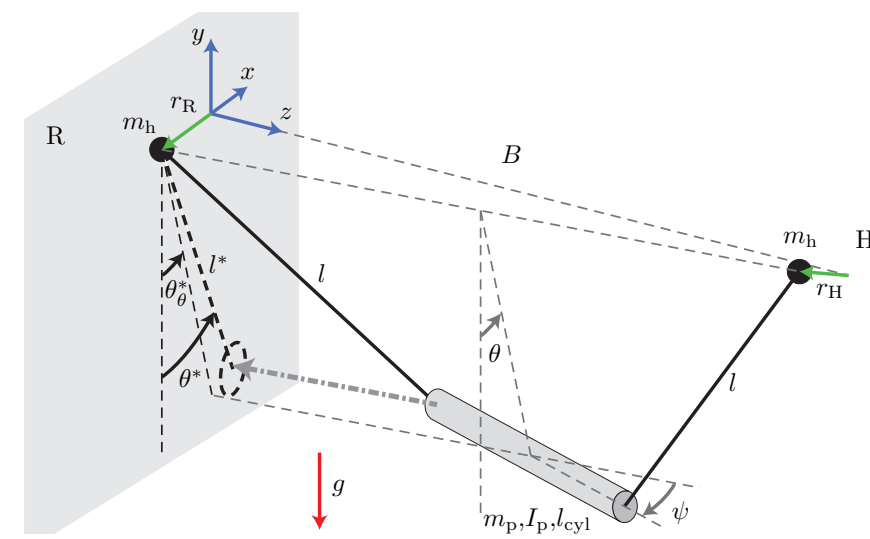

Fig. 2. T-pendulum setup: cylindrical pendulum object of mass $m_{\mathrm{p}}$, length $l_{\text {cyl }}$ and inertia $I_{\mathrm{p}}$ actuated by a human $\mathrm{H}$ and a robot $\mathrm{R}$ through the handle masses $m_{\mathrm{h}}$.

distance between the two handles in their initial configuration is $B$. The position of the human and robot handles, with respect to their initial positions, are given by the vectors $\boldsymbol{r}_{\mathrm{H}}$ and $\boldsymbol{r}_{\mathrm{R}}$, respectively. As in [8], we restrict the handle on the robot side $\mathrm{R}$ to movements in the $x$-direction in a limited range for simplicity and as a minimum requirement. The human handle movement is limited to the $x y$-plane. Due to the restrictions on the handle motion, the pendulum swings predominantly in two modes, captured by the deflection angles $\theta$ and $\psi$. Similar to [8], we base our control on a projection of the pendulum into the $x y$-plane on the robot side R, as indicated by the dash-dotted arrow in Fig. 2 . The projection results in a simple pendulum of length $l^{*}$ and deflection angle $\theta^{*}$. For a deflection angle $\psi=0$ and handles $m_{\mathrm{h}}$ in their initial configuration, the desired deflection angle and their projections are equal $\theta=\theta^{*}=\theta_{\theta}^{*}$. When the two partners move independently, the desired deflection angle does only approximately relate to the projected angles $\theta \approx \theta^{*}=\theta_{\theta}^{*}$ and the initial handle distance $B$ and the projected length $l^{*}$ change. For a deflection angle $\psi \neq 0$ the projected angle $\theta^{*}$ deviates from the deflection angle $\theta \approx \theta_{\theta}^{*}$ representing the desired mode of oscillation. We neglect the change of the initial handle distance $B$ due to $B \gg\left\|\boldsymbol{r}_{\mathrm{H} / \mathrm{R}}\right\|_{2}$ and further assume a constant projected length $l^{*}$, calculated based on the initial configuration.

The v-shaped pendulum investigated in [8] is obtained for $l_{\text {cyl }}=0$. The v-pendulum represents a simplified case of the t-pendulum discussed here, as it only swings in the desired mode represented by $\theta$.

\section{B. Problem statement}

This paper investigates the problem of cooperatively swinging up the complex pendulum-like object displayed in Fig. 2 to a desired energy level $V_{\theta}^{\mathrm{d}}$, represented by a desired maximum deflection angle $\theta_{V}^{\mathrm{d}}$. The complexity of the mechanism results in two possible swinging modes, represented by the deflection angles $\theta$ and $\psi$. We only want to excite the mode related to $\theta$, while simultaneously damping $\psi$. The under-actuated mechanism is controlled through acceleration of two handle masses $m_{\mathrm{h}}$ by two partners, a robot $\mathrm{R}$ and a human $\mathrm{H}: \boldsymbol{u}=\left[\begin{array}{ll}\ddot{\boldsymbol{r}}_{\mathrm{R}} & \ddot{\boldsymbol{r}}_{\mathrm{H}}\end{array}\right]^{T}$. The only controllable input is the robot's acceleration, which we restrict to the $x$-direction (Fig. 2) $u_{\mathrm{R}}=\ddot{r}_{\mathrm{R}, x}$. The robot has to use the forces applied at its end effector $\boldsymbol{F}_{\mathrm{R}}$ to infer the energy content of the two modes $V_{\theta}$ and $V_{\psi}$. Thus, we are looking for a control law

$$
\begin{gathered}
u_{\mathrm{R}}=\ddot{r}_{\mathrm{R}, x}=f\left(\boldsymbol{F}_{\mathrm{R}}\right) \\
\text { with }\left|V_{\theta}^{\mathrm{d}}-V_{\theta}\left(t>T_{\mathrm{s}}\right)\right| \leq \epsilon_{\theta} \\
\text { and }\left|0-V_{\psi}\left(t>T_{\mathrm{s}}\right)\right| \leq \epsilon_{\psi} \quad \text { for } 0<T_{\mathrm{s}}<\infty,
\end{gathered}
$$

where $T_{\mathrm{S}}$ is the system settling time. Hence, we require the energy errors to stay within the ranges $\epsilon_{\theta / \psi}$ the latest after $t=T_{\mathrm{s}}$.

The task goal (3) is known to both partners. For the task goal (2), we distinguish the cases of a robotic leader and a robotic follower based on whether the desired energy $V_{\theta}^{\mathrm{d}}$ is known or unknown to the robot in advance, respectively. Based on the applied force $\boldsymbol{F}_{\mathrm{R}}$ a robotic follower has to estimate the human's intention: increase, hold, or decrease the current energy level $V_{\theta}$ in order to actively contribute to the task goal $V_{\theta}^{\mathrm{d}}$.

\section{PROJECTION ONTO SIMPLE PENDULUM}

Our control approach relies on a projection of the complex pendulum onto an abstracted simple pendulum with twosided actuation.

\section{A. Equation of motion}

The equation of motion that describes the deflection angle $\vartheta$ of a lossless simple pendulum of length $l^{*}$ and whose pivot is accelerated with $\ddot{r}_{x}$ is given by

$$
\ddot{\vartheta}=-\frac{g}{l^{*}} \sin (\vartheta)-\frac{1}{l^{*}} \cos (\vartheta) \ddot{r}_{x} .
$$

The t-pendulum is actuated on two sides, with equal influence of both partners. Whereas the human can move in the $x y$-plane in the virtual reality experiment (Sec. VI), we assume for the following derivations that also the human is limited to move in $x$-direction $\ddot{\boldsymbol{r}}_{\mathrm{R} / \mathrm{H}}=\left[\begin{array}{lll}\ddot{r}_{\mathrm{R} / \mathrm{H}, x} & 0 & 0\end{array}\right]^{T}$. By replacing the acceleration $\ddot{r}_{x}$ by the mean of the accelerations provided by the robot $\ddot{r}_{\mathrm{R}, x}$ and the human $\ddot{r}_{\mathrm{H}, x}$, we obtain the following equation of motion for an abstract simple pendulum with two-sided actuation

$$
\ddot{\vartheta}=-\frac{g}{l^{*}} \sin (\vartheta)-\frac{1}{l^{*}} \cos (\vartheta) \frac{\ddot{r}_{\mathrm{R}, x}+\ddot{r}_{\mathrm{H}, x}}{2} .
$$

This abstraction is a strong simplification, but forms the basis of the presented controllers: It allows us to estimate the energy injected by the human partner, as needed in Sec. IV.

\section{B. Pendulum energy}

The energy of the pendulum with respect to the $\vartheta$-oscillation disregarding the energy induced by the handle motion $\left(\dot{r}_{\mathrm{R}, x}=\dot{r}_{\mathrm{H}, x}=0\right)$ [9] is

$$
V_{\vartheta}=\frac{1}{2} m_{\mathrm{p}} l^{* 2} \dot{\vartheta}^{2}+m_{\mathrm{p}} g l^{*}(1-\cos (\vartheta)) .
$$

Insertion of (5) into the time derivative of (6) yields

$\dot{V}_{\vartheta}=\dot{V}_{\vartheta, \mathrm{R}}+\dot{V}_{\vartheta, \mathrm{H}}=-\frac{1}{2} m_{\mathrm{p}} l^{*} \dot{\vartheta} \cos (\vartheta)\left(\ddot{r}_{\mathrm{R}, x}+\ddot{r}_{\mathrm{H}, x}\right)$. 


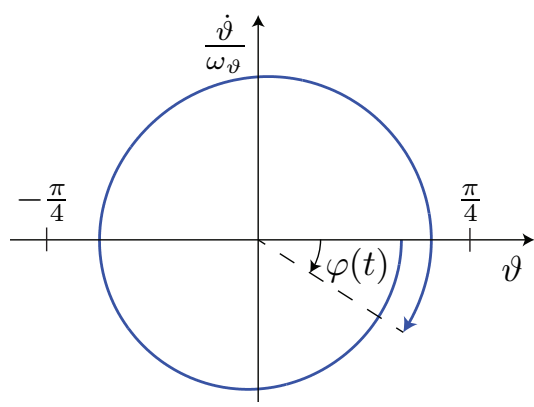

Fig. 3. Section of the phase portrait of a simple pendulum during swing-up.

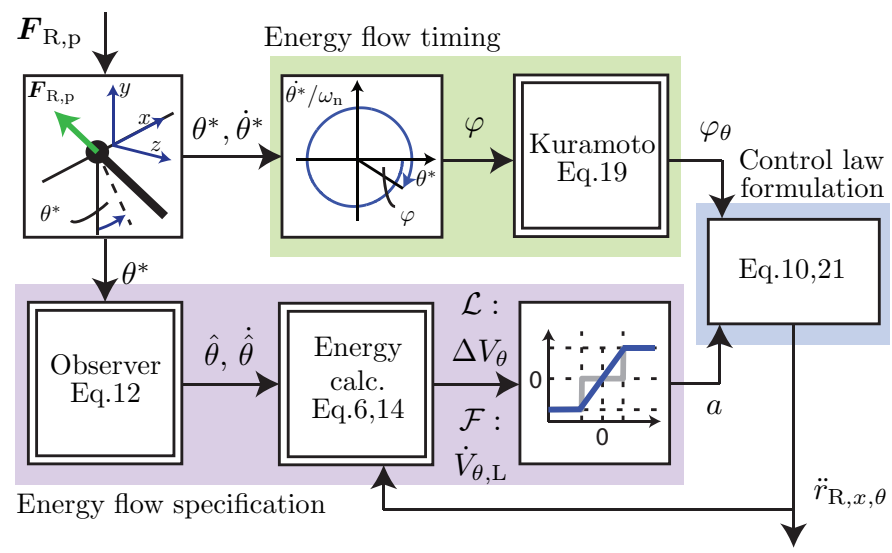

Fig. 4. Control structure for excitation of the $\theta$-oscillation grouped into energy flow specification, energy flow timing and control law formulation.

\section{Natural frequency and phase angle}

For small angles $\vartheta$ the natural frequency of a simple pendulum is approximately $\omega_{\vartheta}=\sqrt{\frac{g}{l^{*}}}$. The actual natural frequency $\omega_{\vartheta}^{\prime}$ of the nonlinear simple pendulum is not constant, but decreases with higher energy contained in the pendulum. The natural frequency $\omega_{\vartheta}^{\prime}$ approximately relates to the phase angle $\varphi$ [9]

$$
\varphi(t) \approx \omega_{\vartheta}^{\prime} t+\varphi_{0} .
$$

Figure 3 shows a phase portrait with inscribed phase angle

$$
\varphi=\operatorname{atan} 2\left(-\frac{\dot{\vartheta}}{\omega_{\vartheta}}, \vartheta\right) .
$$

Calculation of the phase angle $\varphi$ allows us to excite the pendulum close to its natural frequency $\omega_{\vartheta}^{\prime}$.

\section{SWING-UP CONTROLLER BASED ON EXCITATION CLOSE TO NATURAL FREQUENCY}

The cooperative swing-up control approach presented in [8] is based on the swing-up controller of [9] for a simple linearly accelerated pendulum. Here, we extend this approach to swing up more complex pendulums. The double-framed blocks in Fig. 4 represent parts within the controller that have been added or changed. For brevity, only the main aspects and modifications are outlined here. For more details see [8].

The control structure in Fig. 4 can be divided into three groups: energy flow specification, energy flow timing and control law formulation. Within energy flow specification the system energy is analyzed in order to specify magnitude and direction of the energy flow to be provided by the robot given by the amplitude factor $a$. The energy flow timing calculates the phase $\varphi_{\theta}$ related to the $\theta$-oscillation, which allows to excite the pendulum close to its natural frequency $\omega_{\theta}^{\prime}$ (see (8)). The control law formulation combines the outputs of energy flow specification $a$ and timing $\varphi_{\theta}$ to compute the desired robot acceleration

$$
\ddot{r}_{\mathrm{R}, x, \theta} \approx a \omega_{\theta}^{\prime 2} \sin \left(\varphi_{\theta}\right)
$$

For the two different cases of a robotic leader and a robotic follower the block energy calculation differs. A robotic leader knows the task goal $V_{\theta}^{\mathrm{d}}(2)$ and thus only needs to estimate the current system energy $V_{\theta}$ contained in the $\theta$-oscillation to decide on an action. A robotic follower does not know the task goal $V_{\theta}^{\mathrm{d}}$. In order to actively contribute to the unknown task goal, the follower should direct its energy flow into the same direction as the human. Thus, the current contribution to the system energy by the human leader $\dot{V}_{\theta, \mathrm{L}}$ has to be estimated.

The only feedback the robot receives is its end effector force $\boldsymbol{F}_{\mathrm{R}}$. The projected deflection angle $\theta^{*}$ is obtained from

$$
\theta^{*}=\arctan \left(\frac{-F_{\mathrm{R}, \mathrm{p}, x}}{F_{\mathrm{R}, \mathrm{p}, y}}\right),
$$

where the force $\boldsymbol{F}_{\mathrm{R}, \mathrm{p}}$ exerted on the pendulum results from the measurable end effector force $\boldsymbol{F}_{\mathrm{R}}$ by dynamically compensating for the force caused by the handle mass $m_{\mathrm{h}}$.

As the basis of the control approach presented in this paper, the abstract two-sided actuated simple pendulum (Sec. III) is applied to the t-pendulum of Fig. 2 by replacing $\vartheta$ with $\theta \approx \theta_{\theta}^{*}$. An observer and a Kuramoto model [13] based on the abstract simple pendulum extract the mode of oscillation related to $\theta$ from the measured projection angle $\theta^{*}(11)$.

\section{A. Energy flow specification}

1) Nonlinear observer: Our control goal one as defined in (2) is to reach a desired energy level $V_{\theta}^{\mathrm{d}}$ and track it. In order to examine the current system energy contained in the desired oscillation $V_{\theta}$, the projected deflection angle $\theta_{\theta}^{*}$ has to be estimated (see Fig. 2). For this purpose we design a nonlinear observer

$$
\dot{\hat{\boldsymbol{\theta}}}=\left[\begin{array}{c}
\dot{\hat{\theta}} \\
-\frac{g}{l^{*}} \sin (\hat{\theta})
\end{array}\right]+\boldsymbol{L}\left(\theta^{*}-y\right) \quad y=\left[\begin{array}{ll}
1 & 0
\end{array}\right] \hat{\boldsymbol{\theta}}
$$

with state vector $\hat{\boldsymbol{\theta}}=\left[\begin{array}{cc}\hat{\theta} & \dot{\hat{\theta}}\end{array}\right]^{T} \approx\left[\begin{array}{cc}\theta_{\theta}^{*} & \dot{\theta}_{\theta}^{*}\end{array}\right]^{T}$. The observer models the abstract simple pendulum (5), and is coupled to the actual t-pendulum through the term $\boldsymbol{L}\left(\theta^{*}-y\right)$.

The proposed observer differs from the ones that can be found in literature [14]. We do not try to capture the complete complex t-pendulum, but we want to extract the mode of oscillation related to $\theta$. Basically, the measurable projection angle $\theta^{*}$ is an input signal, which includes the oscillation $\psi$ as a disturbance. With the observer we want to filter out the disturbance source $\psi$ to extract the deflection angle $\theta_{\theta}^{*}$, which is related to the $\theta$-oscillation of natural frequency $\omega_{\theta}^{\prime}$. 
From a linearization of (12) around the stable equilibrium point $\hat{\boldsymbol{\theta}}=\mathbf{0}$ we find observer gains $\boldsymbol{L}=\left[\begin{array}{ll}k \omega_{\theta} & 0\end{array}\right]^{T}$ with $k>0$ to extract the mode of oscillation related to $\theta$. All frequency components of the observer input $\theta^{*}$ are damped, except the ones close to the natural frequency $\omega_{\theta}$ of the linearized pendulum. Higher $k$-values result in a lower damping but in a faster observer behavior. Based on simulation, we found $k=1$ to be a good compromise between damping of unwanted frequencies $\omega \neq \omega_{\theta}$ and a sufficiently fast system response. Obviously, better observer performance is to be expected for a greater difference between the natural frequencies of the $\theta$ - and the $\psi$-oscillations.

2) Leader $\mathcal{L}$ : The leader estimates the current energy of the system $V_{\theta}$ from (6) using the observer output $\hat{\boldsymbol{\theta}}$.

As in [8], the amplitude factor $a$ is chosen to increase linearly with the energy error $\Delta V_{\theta}=V_{\theta}^{\mathrm{d}}-V_{\theta}$ and a slope of $\frac{a_{\mathrm{L}, \theta}}{b_{\mathrm{L}, \theta}}$ while being saturated at $\pm a_{\mathrm{L}, \theta}$

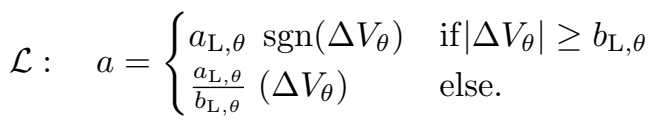

Energy is injected into the system for $a>0$ and released from the system for $a<0$.

3) Follower $\mathcal{F}$ : The follower estimates the leader's energy flow to the system $\dot{V}_{\theta, \mathrm{L}}$ by subtracting its own energy flow $\dot{V}_{\theta, \mathrm{F}}(7)$ from the total change in system energy $\dot{V}_{\theta}$

$$
\dot{V}_{\theta, \mathrm{L}}=\dot{V}_{\theta}-\dot{V}_{\theta, \mathrm{F}} \text {. }
$$

The overall change in energy $\dot{V}_{\theta}$ is calculated by differentiating the system energy $V_{\theta}(6)$ with the following filter

$$
\dot{V}_{\theta}(s)=\frac{s}{T_{\mathrm{F}}^{2} s^{2}+2 T_{\mathrm{F}} s+1} V_{\theta}(s) .
$$

This filter acts as a differentiator approximately up to the frequency $\omega=\frac{1}{T_{F}}$ and damps higher frequencies than $\omega=\frac{1}{T_{F}}$. The robot's energy flow $\dot{V}_{\theta, \mathrm{F}}$ is filtered with

$$
G_{\mathrm{f}}(s)=\frac{1}{T_{\mathrm{F}}^{2} s^{2}+2 T_{\mathrm{F}} s+1} .
$$

As in [8], the estimated leader energy flow $\dot{V}_{\theta, \mathrm{L}}$ is mapped to three discrete values $a_{\mathrm{dis}}=\left\{-a_{\mathrm{F}}, 0, a_{\mathrm{F}}\right\}$ as follows

$$
\mathcal{F}: \quad a_{\mathrm{dis}}= \begin{cases}a_{\mathrm{F}, \theta} \operatorname{sgn}\left(\dot{V}_{\theta, \mathrm{L}}\right) & \text { if }\left|\dot{V}_{\theta, \mathrm{L}}\right| \geq b_{\mathrm{F}, \theta} \\ 0 & \text { else. }\end{cases}
$$

In contrast to the leader (13), a neutral zone is defined by $b_{\mathrm{F}, \theta}$ instead of a ramp connecting the two maximum values $\pm a_{\mathrm{L} / \mathrm{F}, \theta}$. Jumps in the acceleration of the end effector $\ddot{r}_{\mathrm{R}, x, \theta}$ are eliminated through ramps with blending time constant $\tau_{\mathrm{F}}$ [3]

$$
\dot{a}=\tau_{\mathrm{F}} \operatorname{sgn}\left(a_{\mathrm{dis}}-a\right) .
$$

\section{B. Energy flow timing}

For an undesired oscillation $\psi \neq 0$, the phase angle $\varphi$ obtained from the measurable projected deflection angle $\theta^{*}$ (9) does not relate well to the natural frequency of the $\theta$-oscillation we want to excite. In order to extract the phase angle $\varphi_{\theta}$ related to the desired oscillation, we implement a Kuramoto model [13]

$$
\dot{\varphi}_{\theta}=\omega_{\theta}+\kappa \sin \left(\varphi-\varphi_{\theta}\right) .
$$

The Kuramoto oscillator of frequency $\omega_{\theta}$ synchronizes its phase angle $\varphi_{\theta}$ to the t-pendulum through the coupling term $\kappa \sin \left(\varphi-\varphi_{\theta}\right)$ with coupling gain $\kappa$. As for the observer, better performance of the Kuramoto model is to be expected for a greater difference between the natural frequencies of the $\theta$ - and the $\psi$-oscillations.

\section{Control law formulation}

For our case of a position controlled robot, we design a trajectory $r_{\mathrm{R}, x, \theta}$, which complies with the acceleration $\ddot{r}_{\mathrm{R}, x, \theta}$ in (10). As in [8] we use the approach presented in [9] to compute the final trajectory $r_{\mathrm{R}, x, \theta}$. For brevity, we only give a summary here. For details see [8] and [9]. The phase angle $\varphi_{\theta}$ and the amplitude factor $a$ are combined to compute a reference trajectory $r_{\mathrm{R}, x, \theta}^{\mathrm{d}}$, which is fed through a second order transfer function $G_{\theta}(j \omega)$ to filter out higher frequencies than the approximated natural frequency $\omega_{\theta}$

$$
G_{\theta}(j \omega)=\frac{\left(\frac{\omega_{\theta}}{c_{0}}\right)^{2}}{(j \omega)^{2}+2 \zeta \frac{\omega_{\theta}}{c_{0}}(j \omega)+\left(\frac{\omega_{\theta}}{c_{0}}\right)^{2}},
$$

where $c_{0}$ and $\zeta$ are design variables. The resulting final trajectory $r_{\mathrm{R}, x, \theta}$ is approximately

$$
\begin{array}{r}
r_{\mathrm{R}, x, \theta} \simeq-a \frac{\left|G_{\theta}\left(j \omega_{\theta}^{\prime}\right)\right|}{\left|G_{\theta}\left(j \omega_{\theta}\right)\right|} \sin \left(\varphi_{\theta}+\angle G_{\theta}\left(j \omega_{\theta}\right)-\angle G_{\theta}\left(j \omega_{\theta}^{\prime}\right)\right) \\
\approx-a \sin \left(\varphi_{\theta}\right), \quad(21)
\end{array}
$$

where $\left|G_{\theta}\left(j \omega_{\theta}^{\prime}\right)\right|$ and $\angle G_{\theta}\left(j \omega_{\theta}^{\prime}\right)$ are the amplitude and phase shift caused by the filtering. By using the approximated amplitude and phase shift $\left|G_{\theta}\left(j \omega_{\theta}\right)\right|$ and $\angle G_{\theta}\left(j \omega_{\theta}\right)$ within the reference trajectory, we can approximately compensate for the amplitude and phase shift caused by the filtering at the desired natural frequency $\omega_{\theta}^{\prime}$.

Time derivation of (21) yields the control law (1) to fulfill (2) $u_{\mathrm{R}, \theta}=\ddot{r}_{\mathrm{R}, x, \theta}\left(\boldsymbol{F}_{\mathrm{R}}\right) \approx a\left(\boldsymbol{F}_{\mathrm{R}}\right) \omega_{\theta}^{\prime 2} \sin \left(\varphi_{\theta}\left(\boldsymbol{F}_{\mathrm{R}}\right)\right)$.

\section{Damping of undesired $\psi$-oscillation}

By controlling the robot end effector to track $r_{\mathrm{R}, x, \theta}$ of (21), the energy stored in the $\theta$-oscillation is brought close to the task goal $V_{\theta}^{\mathrm{d}}$ as formulated in (2). The $\psi$-oscillation is ignored, in contrast to our second control goal formulated in (3). In order to decrease the energy stored in the $\psi$-oscillation $V_{\psi}$, we extract the part of the projected angle $\theta_{\psi}^{*}$ and angular velocity $\dot{\theta}_{\psi}^{*}$ that is caused by the undesired $\psi$-oscillation (see Fig. 2)

$$
\left[\begin{array}{c}
\theta_{\psi}^{*} \\
\dot{\theta}_{\psi}^{*}
\end{array}\right]=\left[\begin{array}{c}
\theta^{*} \\
\dot{\theta}^{*}
\end{array}\right]-\left[\begin{array}{c}
\theta_{\theta}^{*} \\
\dot{\theta}_{\theta}^{*}
\end{array}\right]
$$

The observer output $\hat{\boldsymbol{\theta}}$ (12) is used as an approximation for $\left[\begin{array}{ll}\theta_{\theta}^{*} & \dot{\theta}_{\theta}^{*}\end{array}\right]^{T}$.

Together with an approximation for the natural frequency $\omega_{\psi}$ obtained from simulation, the phase angle $\varphi_{\psi}$ is calculated (9). 
For the energy flow specification, the pseudo-energy $V_{\psi}$ is calculated using (22) in (6) and the filter (16). The same mapping, but with different parameterization, from energy error $\Delta V_{\psi}=0-V_{\psi}$ to amplitude factor $a$ is used as for the leader exciting the $\theta$-oscillation (13)

$$
\mathcal{L}_{\psi}: \quad a= \begin{cases}a_{\mathrm{L}, \psi} \operatorname{sgn}\left(\Delta V_{\psi}\right) & \text { if }\left|\Delta V_{\psi}\right| \geq b_{\mathrm{L}, \psi} \\ \frac{a_{\mathrm{L}, \psi}}{b_{\mathrm{L}, \psi}}\left(\Delta V_{\psi}\right) & \text { else. }\end{cases}
$$

Control goal (3) is achieved by controlling the robot to track

$$
\begin{array}{r}
r_{\mathrm{R}, x, \psi} \simeq-a \frac{\left|G_{\psi}\left(j \omega_{\psi}^{\prime}\right)\right|}{\left|G_{\psi}\left(j \omega_{\psi}\right)\right|} \sin \left(\varphi_{\psi}+\angle G_{\psi}\left(j \omega_{\psi}\right)-\angle G_{\psi}\left(j \omega_{\psi}^{\prime}\right)\right) \\
\approx-a \sin \left(\varphi_{\psi}\right) .
\end{array}
$$

Note that the same approach as in (21) based on [9] is applied to obtain the robot trajectory $r_{\mathrm{R}, x, \psi}$, but with a second order transfer function $G_{\psi}(j \omega)$ that filters out higher frequencies than the approximated natural frequency $\omega_{\psi}$.

The control law (1) damping the $\psi$-oscillation (3) results to $u_{\mathrm{R}, \psi}=\ddot{r}_{\mathrm{R}, x, \psi}\left(\boldsymbol{F}_{\mathrm{R}}\right) \approx a\left(\boldsymbol{F}_{\mathrm{R}}\right) \omega_{\theta}^{\prime 2} \sin \left(\varphi_{\psi}\left(\boldsymbol{F}_{\mathrm{R}}\right)\right)$. The $\theta$-excitation and $\psi$-damping are combined on position level by summation of (21) and (24)

$$
r_{\mathrm{R}, x}=r_{\mathrm{R}, x, \theta}+r_{\mathrm{R}, x, \psi} .
$$

\section{SIMULATION BASED DISCUSSION}

To investigate the controller performance, we simulated a robot follower cooperating with a robot leader.

\section{A. Software Implementation}

The simulation is implemented using MATLAB/Simulink ${ }^{\circledR}$. The t-pendulum is modeled via rigid bodies connected through unconstrained spherical joints using the SimMechanics toolbox. Due to stability issues, the ropes are modeled as masses $m_{\mathrm{r}}=1 \mathrm{~kg}$ of inertia $\boldsymbol{I}_{\mathrm{r}}=\operatorname{diag}\left\{I_{\mathrm{r}}\right\}$ with $I_{\mathrm{r}}=0.0005 \mathrm{kgm}^{2}$ located at the connection to the cylindrical object. The cylindrical mass in turn is reduced to $m_{\mathrm{cyl}}=3 \mathrm{~kg}$, such that a resulting pendulum mass $m_{\mathrm{p}}=5 \mathrm{~kg}$ is obtained. The t-pendulum dimensions are $B=1.32 \mathrm{~m}$ and $l=0.74 \mathrm{~m}$, with the pendulum object modeled as a cylindrical mass $m_{\mathrm{cyl}}$ of length $l_{\text {cyl }}=0.5 \mathrm{~m}$ and radius $r_{\text {cyl }}=0.05 \mathrm{~m}$ with uniform mass distribution. The translational and rotational motion of the cylindrical mass are damped with viscous damping $d_{\mathrm{cyl}, \mathrm{t}}=0.1 \mathrm{Ns} / \mathrm{m}$ and $d_{\mathrm{cyl}, \mathrm{r}}=0.02 \mathrm{Nms} / \mathrm{rad}$ in all directions, respectively. The mechanism geometry leads to natural frequencies $\omega_{\theta}=\sqrt{\frac{g}{l^{*}}}=4 \mathrm{rad} / \mathrm{s}$ and $\omega_{\psi} \approx 8 \mathrm{rad} / \mathrm{s}$, with $\omega_{\psi}$ obtained from simulation.

The control parameters used for the pendulum simulation as well as the virtual reality experiment in Sec. VI are listed in Table I.

\section{B. Simulation Experiment and Measures}

The control parameters are tuned for initial deflection angles $\theta_{0,1}=\theta(t=0)=10^{\circ}$ and $\psi_{0,1}=\psi(t=0)=0^{\circ}$ to achieve approximately a follower energy contribution of $50 \%$ with low overshoot. The desired energy level $V_{\theta}^{\mathrm{d}}$ is set to a maximum deflection angle $\theta_{V}^{\mathrm{d}}=\frac{\pi}{4}$.
TABLE I

CONTROL PARAMETERS

\begin{tabular}{cccccc}
$a_{\mathrm{L}, \theta}[\mathrm{m}]$ & $b_{\mathrm{L}, \theta}[\mathrm{J}]$ & $a_{\mathrm{F}, \theta}[\mathrm{m}]$ & $b_{\mathrm{F}, \theta}[\mathrm{W}]$ & $a_{\mathrm{L}, \psi}[\mathrm{m}]$ & $b_{\mathrm{L}, \psi}[\mathrm{J}]$ \\
\hline 0.0405 & 3.9 & 0.028 & 0.2 & 0.0135 & 0.2 \\
& & & & & \\
$L_{1}$ & $T_{\mathrm{F}}$ & $\kappa$ & $c_{0}$ & $\zeta$ & $\tau_{\mathrm{F}}[\mathrm{s}]$ \\
\hline$\omega_{\theta}$ & $\frac{1.5}{\omega_{\theta}}$ & $\omega_{\theta}$ & 0.9 & 1.2 & 1
\end{tabular}

We analyze the controller performance and effort sharing for different initial deflection angles with increasing disturbance $\psi$. For the performance analysis we compute the settling time $T_{\mathrm{s}}$ as the amount of time needed until the system energy $V$ stays within $2 \%$ of the steadystate value of the system energy $V_{\mathrm{ss}}$ and the steady-state error $e_{\mathrm{ss}}=V_{\theta}^{\mathrm{d}}-V_{\mathrm{ss}}$ [15]. Furthermore, we analyze effort sharing based on the relative follower contribution $R C_{\mathrm{F}}$. The relative follower contribution $R C_{\mathrm{F}}$ is the energy input of the follower $V_{\mathrm{F}}$ in relation to the energy input of both agents within $\left[t_{0}, t_{0}+T_{\mathrm{s}}\right]$

$$
R C_{\mathrm{F}}=\frac{V_{\mathrm{F}}\left(t_{0}+T_{\mathrm{s}}\right)-V_{\mathrm{F}}\left(t_{0}\right)}{V_{\mathrm{F}}\left(t_{0}+T_{\mathrm{s}}\right)-V_{\mathrm{F}}\left(t_{0}\right)+V_{\mathrm{L}}\left(t_{0}+T_{\mathrm{S}}\right)-V_{\mathrm{L}}\left(t_{0}\right)},
$$

with the two controllers being engaged at $t_{0}=3 \mathrm{~s}$.

For brevity, we focus on the simulation results for $\theta_{0,2}=5.7^{\circ}$ and $\psi_{0,2}=19.4^{\circ}$. Figure 5(a) shows energies over time. The energies injected into the t-pendulum by the robot leader $V_{\mathrm{L}}$ and the robot follower $V_{\mathrm{F}}$ add up to the total energy contained in the system $V$, an energy loss due to damping and the initial system energy. The energy contained in the $\theta$-oscillation $V_{\theta}$ is calculated according to (6). Figure 5(b) shows the angles $\theta$ and $\psi$ in relation to the desired maximum deflection angle $\theta_{V}^{\mathrm{d}}$ over time. The complete energy flow caused by the leader $\dot{V}_{\mathrm{L}}$ in comparison to the estimated energy flow $\dot{V}_{\theta, \mathrm{L}}$ directed towards the $\theta$-oscillation is displayed in Fig. 5(c). The control parameter $b_{\mathrm{F}, \theta}$ defines the neutral zone that has to be exceeded by $\dot{V}_{\theta, \mathrm{L}}$ to cause the follower to react. Figure 5(d) shows the individual follower trajectories $r_{\mathrm{F}, x, \theta}$ and $r_{\mathrm{F}, x, \psi}$ and their combination $r_{\mathrm{F}, x}$.

\section{Discussion}

1) Analysis of task performance: We first analyze the task performance according to the achievement of our control goals as stated in (2) and (3) for the two pairs of initial conditions $\theta_{0,1}=10^{\circ}, \psi_{0,1}=0^{\circ}$ and $\theta_{0,2}=5.7^{\circ}$, $\psi_{0,2}=19.4^{\circ}$.

For both pairs of initial conditions, the system energy $V$ converges to a steady-state value $V_{\mathrm{ss}}$ close to the desired system energy $V_{\theta}^{\mathrm{d}}$ with small steady-state errors $e_{\mathrm{ss}, 1}=1.7 \%$ and $e_{\mathrm{ss}, 2}=1.8 \%$. The settling time increases from $T_{\mathrm{s}, 1}=15.5 \mathrm{~s}$ to $T_{\mathrm{s}, 2}=19.3 \mathrm{~s}$. From various simulations with different initial conditions, we found the initial disturbances $\psi_{0}$ to cause the increase in settling time.

According to our control goals (2) and (3), the steadystate energy $V_{\mathrm{ss}}$ has to primarily consist of energy in the $\theta$-oscillation. The energy contained in the $\psi$-oscillation 

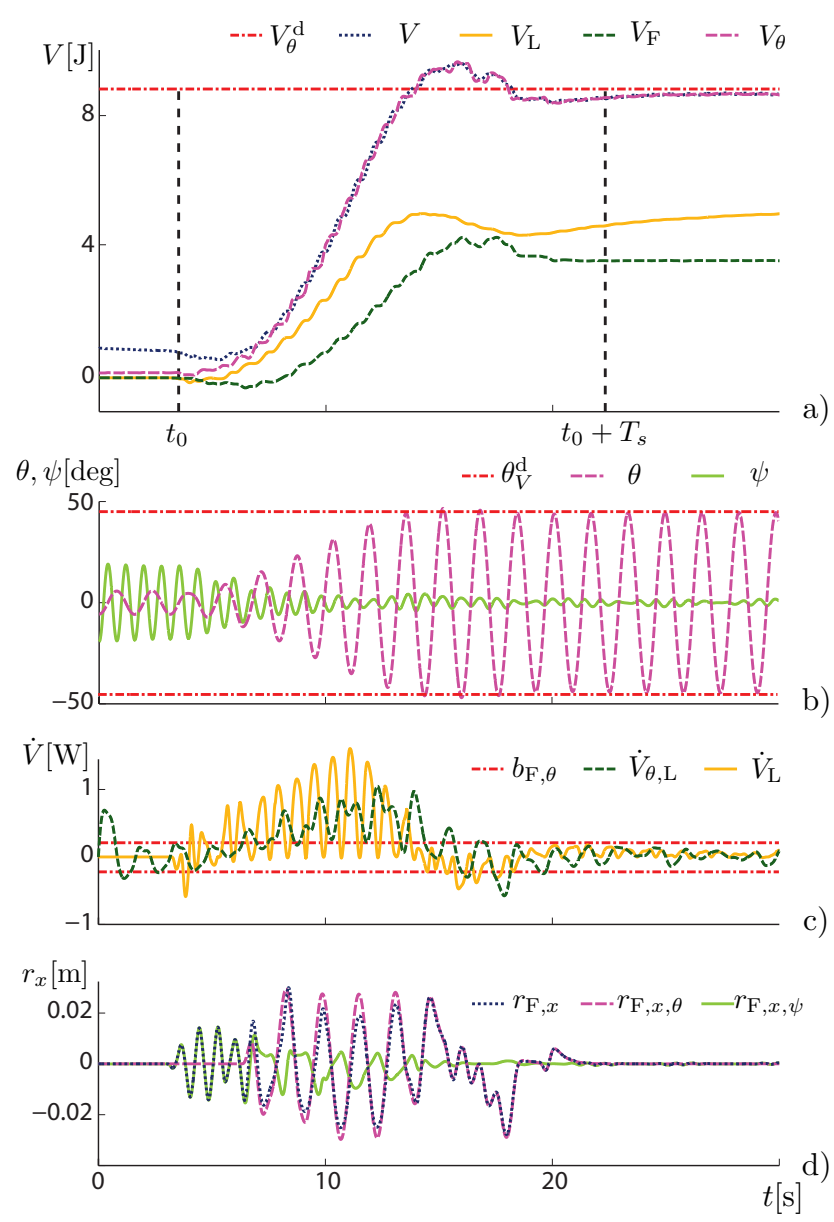

Fig. 5. Example trial of a robot follower cooperating with a robot leader in simulation for initial conditions $\theta_{0,2}=5.7^{\circ}, \psi_{0,2}=19.4^{\circ}$ and a desired energy specified by $\theta_{V}^{\mathrm{d}}=\frac{\pi}{4}$ : (a) energies $V$, (b) angles $\theta$ and $\psi$, (c) actual energy flow of leader $\dot{V}_{\mathrm{L}}$ and estimation $\dot{V}_{\theta, \mathrm{L}}$ directed towards the $\theta$-oscillation, (d) composition of follower trajectory $r_{x}$.

can be approximated by $V_{\psi} \approx V-V_{\theta}$. Due to the initial disturbance in $\psi_{0,2}=19.4^{\circ}$, most of the initial system energy is contained in the $\psi$-oscillation (see Fig. 5(a)). After $t_{0}=3 \mathrm{~s}$, both robot controllers start to decrease the energy contained in the $\psi$-oscillation, with first the robot leader and later also the robot follower simultaneously injecting energy into the $\theta$-oscillation (see Fig. 5(a) and (b)). As a consequence of the decaying energy contained in the $\psi$-oscillation, the energy contained the $\theta$-oscillation $V_{\theta}$ approaches the overall system energy $V$. The energies $V_{\theta}$ and $V$ never completely overlap due to the approximation made in the energy computation based on the abstract simple pendulum (6) and a small remaining $\psi$-oscillation of $|\psi|_{\max }=2^{\circ}$.

For an initial deflection $\psi_{0,1}=0^{\circ}$, the initial system energy is solely contained in the $\theta$-oscillation. However, the $\theta$-excitation causes an undesired $\psi$-oscillation, which has to be actively damped.

Summing up, we achieve our control goals as stated in (2) and (3) with the presented control approach with and without a significant initial disturbance in $\psi$.
2) Analysis of effort sharing: The follower controller strongly relies on the abstract simple pendulum to estimate the energy flow $\dot{V}_{\theta, \mathrm{L}}$ caused by the leader and directed towards the $\theta$-oscillation. Figure 5 shows that the estimated energy flow $\dot{V}_{\theta, \mathrm{L}}$ calculated with (14) follows the overall trend of the actual energy flow $\dot{V}_{\mathrm{L}}$ with a time delay of around $1.2 \mathrm{~s}$ caused by the observer (12) and the filter (15).

Initial deviations of $\dot{V}_{\theta, \mathrm{L}}$ from the leader's actual energy flow $\dot{V}_{\mathrm{L}}=0$ are visible in Figure 5(c). The initial peak is due to the follower's observer not having converged yet and does decay to zero for the first pair of initial conditions with $\psi_{0,1}=0^{\circ}$. The remaining misestimation of $\dot{V}_{\theta, \mathrm{L}}$ for the second pair of initial conditions with $\psi_{0,2}=19.4^{\circ}$ shows that $\psi$-oscillations disturb the estimation of $\dot{V}_{\theta, \mathrm{L}}$. Consequently, the relative follower contribution $R C_{\mathrm{F}}$ decreases from $R C_{\mathrm{F}, 1}=49 \%$ to $R C_{\mathrm{F}, 2}=44 \%$.

Even though the combination of leader and follower controllers are tuned for zero initial disturbance, a wellbalanced effort sharing is achieved for significant initial disturbances $\psi \neq 0^{\circ}$. The results confirm the practicability of the abstract simple pendulum approximation.

3) Combination of $\theta$-excitation and $\psi$-damping: The separate control laws for $\theta$-excitation and $\psi$-damping are combined by a simple summation in (25). Figure 5(d) shows how the two controllers add up to the final trajectory executed by the follower. Initially, the follower exclusively damps the $\psi$-oscillation. At around $t=6 \mathrm{~s}$ the estimation of the leaders energy flow $\dot{V}_{\theta, \mathrm{L}}$ exceeds $b_{\mathrm{F}, \theta}$ (see Fig. 5(c)). Consequently, the trajectory tracked by the follower is a combination of $\theta$-excitation and $\psi$-damping. During $\theta$-excitation the trajectory $r_{\mathrm{F}, x, \psi}$ changes from a dominant frequency of $\omega=\omega_{\psi}$ to $\omega \approx \omega_{\theta}$, but a $\psi$-damping effect remains (see Fig. 5(b) and (d)).

In summary, we are able to effectively damp the undesired $\psi$-oscillation during $\theta$-excitation through a simple summation of the outputs of two separate controllers. The $\psi$-controller contains a number of approximations, like the computation of $\theta_{\psi}^{*}$ (22) used to obtain the pseudoenergy $V_{\psi}$. In face of these approximations, the successful combination of two control goals through summation shows how powerful the swing-up control approach of [9] is.

\section{VIRTUAL REALITY EXPERIMENT}

We conducted a virtual reality experiment to show the transferability of the proposed controller to a setup with a human interaction partner. For brevity, we focus on cooperative swinging of a robot follower interacting with a human leader.

\section{A. Experimental setup}

The experimental setup of [8] was adapted to the tpendulum. Only a short summary is given here. A human cooperatively swings up the t-pendulum with a virtual robotic partner through interaction with a 4 degree of freedom haptic interface. The human may move within the $x y$-plane (Fig. 2), while being restricted to a certain workspace. A force sensor below the handle of the haptic interface measures the 


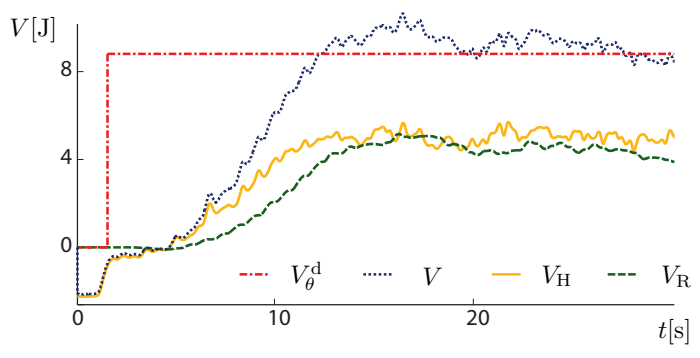

Fig. 6. Energy over time for an example trial of a robot follower cooperating with a human leader in virtual reality.

forces exerted by the human $\boldsymbol{F}_{\mathrm{H}}$. The t-pendulum simulation takes the handle position of the virtual robot $\boldsymbol{r}_{\mathrm{R}}$ and the force exerted by the human $\boldsymbol{F}_{\mathrm{H}}$ as input and generates the force $\boldsymbol{F}_{\mathrm{R}}$ measured by the robot and a human handle position $\boldsymbol{r}_{\mathrm{H}}$, which is tracked by the haptic interface. The virtual reality scene is visualized for the human partner on a screen including a goal sphere marking the desired maximum deflection angle $\theta_{V}^{\mathrm{d}}=\frac{\pi}{4}$ for a human leader. The resulting energies of a sample trial are shown in Fig. 6. The initial angles are both zero $\psi_{0}=\theta_{0}=0$.

\section{B. Discussion}

After an initial lift of the handle, the human leader starts to inject energy into the pendulum (see Fig. 6). The robot follower successfully contributes to the human leader's goal. Due to imprecisions in the human actuation the desired energy level $V_{\theta}^{\mathrm{d}}$ cannot be tracked as accurate as for the simulation (see Fig. 5(a)). The human's actuation together with the $\psi$-damping controller keep the undesired oscillation within $|\psi|_{\max }=4^{\circ}$.

\section{CONCLUSION AND FUTURE WORK}

This paper extends the control approach introduced in [8] to cooperative swinging of a complex pendulum-like object that can swing in two modes. The presented control approach allows to excite one mode up to a desired pendulum energy, while damping the other mode. The desired oscillation of the complex pendulum motion is mapped to an abstract simple pendulum with two-sided actuation. A nonlinear observer and a Kuramoto model based on the abstract simple pendulum are used to extract the desired mode of oscillation to excite it close to its natural frequency. The abstraction to a simple pendulum further allows a robot follower to estimate the energy flow caused by the leading partner, and thus to actively contribute to the leader's task goal. Simulation results confirm that a significant initial disturbance caused by the undesired mode of oscillation can be successfully damped, while the desired mode of oscillation is excited at the same time. A virtual reality experiment shows that the presented control approach can be transferred to a human interaction partner.

To evaluate our work on pendulum-like object swinging, we plan to test the presented control approach in a realistic setup with a real robot. Having investigated swinging of pendulum-like objects, we want to direct our research towards human-robot cooperative swinging of rigid objects, to finally address swinging of semi-rigid objects, e.g. a mattress.

\section{ACKNOWLEDGMENT}

The authors would like to thank Alexander Mörtl, Daniel Althoff, Laith Alkurdi, Sotiris Apostolopoulus and Moritz Stötter for the helpful discussions. This work is supported in part within the ERC Advanced Grant SHRINE Agreement No. 267877 (www. shrine-project.eu).

\section{REFERENCES}

[1] K. Kosuge, H. Yoshida, and T. Fukuda, "Dynamic control for robothuman collaboration," in Proc. 2nd IEEE Int. Workshop on Robot and Human Communication, 1993, pp. 398-401.

[2] B. Corteville, E. Aertbelien, H. Bruyninckx, J. De Schutter, and H. Van Brussel, "Human-inspired robot assistant for fast point-to-point movements," in Proc. IEEE ICRA, 2007, pp. 3639-3644.

[3] A. Mörtl, M. Lawitzky, A. Küçükyılmaz, M. Sezgin, C. Basdogan, and S. Hirche, "The Role of Roles: Physical Cooperation between Humans and Robots," Int. Journal of Robotics Research, vol. 31, no. 13, pp. $1656-1674,2012$

[4] J. Nakanishi, T. Fukuda, and D. Koditschek, "A brachiating robot controller," IEEE Trans. on Robotics and Automation, vol. 16, no. 2, pp. 109-123, 2000.

[5] N. Zoso and C. Gosselin, "Point-to-point motion planning of a parallel 3-dof underactuated cable-suspended robot," in Proc. IEEE ICRA, may 2012, pp. 2325-2330.

[6] C. Kim, K. Yonekura, H. Tsujino, and S. Sugano, "Physical control of the rotation center of an unsupported object rope turning by a humanoid robot," in Proc. 9th IEEE-RAS Int. Conf. on Humanoid Robots, 2009, pp. 148-153.

[7] Y. Maeda, A. Takahashi, T. Hara, and T. Arai, "Human-robot cooperation with mechanical interaction based on rhythm entrainmentrealization of cooperative rope turning," in Proc. IEEE ICRA, vol. 4, 2001, pp. 3477-3482 vol.4.

[8] P. Donner, A. Mörtl, S. Hirche, and M. Buss, "Human-robot cooperative object swinging," in Proc. IEEE ICRA, 2013, pp. 4328-4334.

[9] K. Yoshida, "Swing-up control of an inverted pendulum by energybased methods," in Proc. ACC, vol. 6, 1999, pp. 4045-4047 vol.6.

[10] H. Wang and K. Kosuge, "Control of a robot dancer for enhancing haptic human-robot interaction in waltz," IEEE Transactions on Haptics, vol. 5, no. 3, pp. 264-273, Quarter.

[11] K. Yano and K. Terashima, "Robust liquid container transfer control for complete sloshing suppression," IEEE Transactions on Control Systems Technology, vol. 9, no. 3, pp. 483-493, 2001.

[12] R. J. Full and D. E. Koditschek, "Templates and anchors: neuromechanical hypotheses of legged locomotion on land," Journal of Experimental Biology, vol. 202, no. 23, pp. 3325-3332, 1999.

[13] J. A. Acebrón, L. L. Bonilla, C. J. P. Vicente, F. Ritort, and R. Spigler, "The kuramoto model: A simple paradigm for synchronization phenomena," Reviews of modern physics, vol. 77, no. 1, p. 137, 2005.

[14] B. Walcott, M. Corless, and S. Żak, "Comparative study of non-linear state-observation techniques," Int. Journal of Control, vol. 45, no. 6 , pp. 2109-2132, 1987.

[15] K. J. Astrom and R. M. Murray, Feedback systems: an introduction for scientists and engineers. Princeton university press, 2008. 